\begin{tabular}{|c|c|}
\hline Title & In-situ observation of crystal surfaces by optical microscopy \\
\hline Author(s) & Sazaki, Gen; Nagashima, Ken; Murata, Ken-ichiro; Furukawa, Y oshinori \\
\hline Citation & $\begin{array}{l}\text { Progress in crystal growth and characterization of materials, 62(2), } 408-412 \\
\text { https://doi.org/10.1016 } 1 \text {.pcry sgrow.2016.04.024 }\end{array}$ \\
\hline Issue Date & 2016-06-03 \\
\hline Doc URL & http:/hdl.handle.net/2115/70674 \\
\hline Rights & $\begin{array}{l}\text { O2016, Elsevier. Licensed under the Creative Commons A ttribution-NonCommercial-NoDerivatives } 4.0 \text { International } \\
\text { http://reativecommons.org/icenses/oy-nc-nd/4.0/ }\end{array}$ \\
\hline Rights(URL) & http://creativecommons.org/icenses/by-nc-nd/4.0/ \\
\hline Type & article (author version) \\
\hline File Information & PCGCM62, Sazaki, 2016 - .pdf \\
\hline
\end{tabular}

Instructions for use 


\title{
In-situ observation of crystal surfaces by optical
}

\section{microscopy}

\author{
Gen Sazaki, Ken Nagashima, Ken-ichiro Murata, Yoshinori Furukawa \\ Institute of Low Temperature Science, Hokkaido University \\ N19-W8, Kita-ku, Sapporo 060-0819, Japan
}

\begin{abstract}
In this experimental course, attendees will learn how to obtain useful information about growth processes of crystals using ordinary optical microscopes, which are usually available in laboratories. We will demonstrate how thicknesses of crystals can be estimated from interference colors. We will also show in-situ observations of spiral steps and strain distributions by differential interference contrast microscopy and polarizing microscopy, respectively.
\end{abstract}

Keywords: Optical microscopy; Interference color; Differential interference contrast microscopy; Polarizing microscopy

\section{Introduction}

In many cases, crystals are bounded by flat facets (low index faces), and such crystals grow layer by layer, as illustrated in Fig. $1[1,2]$. We deal with such growth processes. First, we show how to measure thicknesses of plate-like $\mathrm{CdI}_{2}$ crystals utilizing their 
interference colors. Next, we observe bunched spiral growth steps on spiral growth hillocks of $\mathrm{CdI}_{2}$ crystals using a differential interference contrast microscope. Then, we also observe strain distributions around screw dislocations on $\mathrm{SiC}$ crystal surfaces using a polarizing microscope. Through these observations, we hope that attendees realize how ordinary optical microscopes are beneficial for obtaining valuable information about crystal growth. For general information about optical microscopy, see references[3, 4$]$.

\section{Measurement of thicknesses of plate-like crystals using interference colors}

First please remind yourself of the principles behind Newton's rings (Fig. 2A). An illuminating light beam is reflected from two glass-air interfaces. When the difference $R$ in the light paths of two reflected light beams is equal to the integral multiple $i$ of a wavelength $\lambda$, interference of the light occurs. In the case of a monochromatic light beam, with increasing $R$, the interfered light shows repeating dark and light contrasts, as shown in Fig. 2B.

In contrast, in the case of a white light beam consisting of light beams of all visible wavelengths, each light beam with a certain wavelength shows repeating dark and light contrasts, with increasing $R$ : a blue light beam presents dark, blue, dark, blue, and a green light beam depicts dark, green, dark green (Fig. 2B). As a result, the interfered white light shows an "interference color" that is determined by the summation of the interfered light of all visible wavelengths[5, 6]. For example, since the interfered light of zero order $(R \sim 0)$ shows a dark contrast irrespective of wavelength, interfered white 
light presents a black color (Fig. 2C). In contrast, when $R=200-300 \mathrm{~nm}$, all visible wavelengths are in the light contrast conditions. Hence, interfered white light shows a white color. With further increases in $R$, the interference color changes as shown in the color chart in Fig. 2C.

A thin plate-like crystal follows the same principle of the Newton rings. Two light beams reflected from top and bottom surfaces of a plate-like crystal show an interference color. By comparing the interference color of a sample crystal and that shown in the color chart (Fig. 2C), we can evaluate the optical pass difference $R$. Hence, if the refractive index $n$ of a sample plate-like crystal is known, one can obtain the thickness $d$ of the crystal. When $R \geq 1500 \mathrm{~nm}$, the interference color becomes white, showing that one cannot evaluate $R$ from the interference color any more. In other words, if a transparent plate-like crystal shows a certain interference color, $R$ is smaller than $1500 \mathrm{~nm}$.

\section{Observation of spiral growth hillocks using a differential interference contrast}

\section{microscope}

Differential interference contrast (DIC) microscopy provides three-dimensional (3D) contrast using the interference of light [7]. Figure 3 shows a schematic optical path of a DIC microscope of the reflection type. A light beam emitted from a light source is changed into a polarized light beam after passing through a polarizer. Then a Nomarski prism and an objective lens transform the polarized light beam into two parallel light beams whose polarization directions are perpendicular to each other: $\mathrm{O}$ and $\mathrm{E}$ denote 
ordinary and extraordinary rays, respectively. The two parallel light beams are separated by a distance $S$. Since the two parallel light beams have no phase difference, their wave fronts are matched (Fig. 4A). When a convex sample (shown schematically in Fig. 4B) is illuminated by the two parallel light beams, the two reflected light beams have phase distributions according to the convex sample surface (Fig. 4B). Hence, after the two reflected light beams are interfered by passing through an analyzer, the interfered light beam has the phase distribution $(=\mathrm{E}-\mathrm{O})$ shown in Fig. 4C. Depending on this phase distribution, the interference color shown in Fig. 2C appears.

When the optical paths of 0- and E-rays in the Nomarski prism are the same, the zero order interference corresponds to the position of $R=0$ in the color chart (Fig. 2C). Hence, as schematically shown in Fig. 5A, the flat surfaces of the convex object show a black color because of $R=0$. Then the right and left slopes of the convex object (which respectively have negative and positive phase differences (Fig. 4C)) depict a gray color. In contrast, the optical paths of O- and E-rays passing through the prism can be freely changed by shifting the position of the Nomarski prism. Hence, the position of the zero order interference in the interference color chart $\left(\delta^{\prime}=0\right.$ in Fig. $\left.5 \mathrm{~B}\right)$ can be also freely adjusted by this operation. When the position of the zero order interference is adjusted at the position of a gray color, the flat faces of the convex object also show a gray color. In addition, the right and left slopes of the convex object depict blacker and whiter colors than the flat faces, respectively. As a result, we can obtain 3D contrast as if the sample surface is illuminated by a light beam slanted from the left to right direction.

Figure 6 shows a DIC microscopy image of a spiral growth hillock on a tetragonal 
crystal of hen egg-white lysozyme (model protein). By changing the position of the zero order interference, we obtained images as if the crystal surface was illuminated from upper-left (Fig. 6A), above (Fig. 6B) and lower-right (Fig. 6C) directions. In the experimental course, we will observe bunched steps on spiral growth hillocks of $\mathrm{CdI}_{2}$ crystals by DIC microscopy.

\section{Observation of strain distributions in crystals using a polarizing microscope}

By utilizing a polarizing microscope, we can visualize anisotropy in the optical path lengths at a several-nanometer level[5]. Hence, polarizing microscopy is very useful to directly observe strain distributions in crystals.

First we deal with the case in which we observe isotropic samples, such as liquids, amorphous materials and cubic crystals [8]. In this case, the refractive indexes in the two directions ( $a$ - and $c$-directions in Fig. 7A) that are normal to each other are the same. A light beam emitted from a light source is changed into a polarized light beam after passing through a polarizer. When a polarized light beam passes through an isotropic sample, the components of the polarized light in the $a$-and $c$-directions have the same velocity because of the same refractive indexes in these directions. Hence, as shown schematically in Fig. 7A, the direction of the electric field vector of the transmitted polarized light, which is given as the resultant vector of the two components in the $a$ - and $c$-directions, remains in the same direction as that of the illuminating polarized light. No rotation of the polarized light occurs. As a result, the transmitted polarized light cannot pass through the analyzer that is normal to the polarizer. In the 
case of a cubic crystal that is distorted by strain, such distorted regions show bright contrast because of the anisotropy in the region, as explained in the next paragraph.

Next, we deal with anisotropic crystals other than those that are cubic [8]. In this case, the refractive indexes in the two directions ( $a$ - and $c$-directions in Fig. 7B) that are normal to each other are different. Hence, the components of the polarized light in the $a$ - and $c$-directions have different velocities, as schematically shown in Fig. 7B. As a result, the direction of the transmitted polarized light, which is given as the resultant vector of the two components in the $a$ - and $c$-directions, rotates as the polarized light travels in the crystal. Therefore, the transmitted polarized light can pass through the analyzer that is normal to the polarizer. In the case of an anisotropic crystal, after we rotate the sample crystal so that the direction of the transmitted polarized light (given as the resultant vector of the two components in the $a$ - and $c$-directions) becomes normal to the direction of the analyzer (where the whole crystal exhibited a dark contrast), strained regions in the crystal show bright contrast.

Figure 8 present a polarizing microscopy image of a tetragonal crystal of hen egg-white lysozyme (model protein). First we made the polarizer and analyzer normal to each other, and rotated the tetragonal crystal until the whole crystal exhibited a dark contrast. Then we took an image with a long-time exposure (30-60 s). The contrast of the central portion is significantly different from that of the peripheral area. This suggests that the central portion that grew under a higher supersaturation condition in the early stage of the crystallization had a larger amount of strain than the peripheral portion grown under a lower supersaturation condition in the late stage. In the experimental course, we will 
observe strain distributions around dislocations $[9,10]$ in $\mathrm{SiC}$ crystals (provided by Prof. K. Tsukamoto and Dr. K. Murayama).

\section{Summary}

Optical microscopes are often used in science projects in junior-high and high schools. Hence, many people suppose that ordinary optical microscopy is a kind of low-level technology. However, once we fully understand its principles and appropriately adjust the microscopes, we can obtain a large amount of valuable information on crystal growth by ordinary optical microscopy. We hope that attendees of the course come to realize its power.

\section{References}

[1] A.A. Chernov, Modern crystallography III, Springerr-Verlag, Berlin, 1984.

[2] I.V. Markov, Crystal growth for beginners, World Scientific Publishing, Singapore, 1995.

[3] R.C. Gifkins, Optical microscopy of metals, Pitman, London, 1971.

[4] S. Bradbury, An introduction to the optical microscope, Oxford University Press, Oxford, 1989.

[5] N.H. Hartshorne, A. Stuart, Crystals and the polarising microscope, Arnold, London, 1960 .

[6] S. Tsuboi, Henko Kenbikyo (in Japanese), Iwanami, Tokyo, 1959.

[7] D. Murphy, Differential interference contrast microscopy and modulation contrast microscopy, in: Fundamentals of light microscopy and degital imaging, Wiley-Liss, New York, 2001.

[8] P. Gay, Crystal optics, Longman house, Singapore, 1967.

[9] C.Z. Ge, N.B. Ming, K. Tsukamoto, K. Maiwa, I. Sunagawa, Birefringence Images of Screw Dislocations Viewed End-on in Cubic-Crystals, Journal of Applied Physics, 
69 (1991) 7556-7564.

[10] K. Maiwa, K. Tsukamoto, I. Sunagawa, C.Z. Ge, N.B. Ming, Observation of Screw and Mixed Dislocations in Barium Nitrate Crystals by Means of Birefringence and X-Ray Topography, Journal of Crystal Growth, 98 (1989) 590-594. 


\section{Figures and Figure Captions}

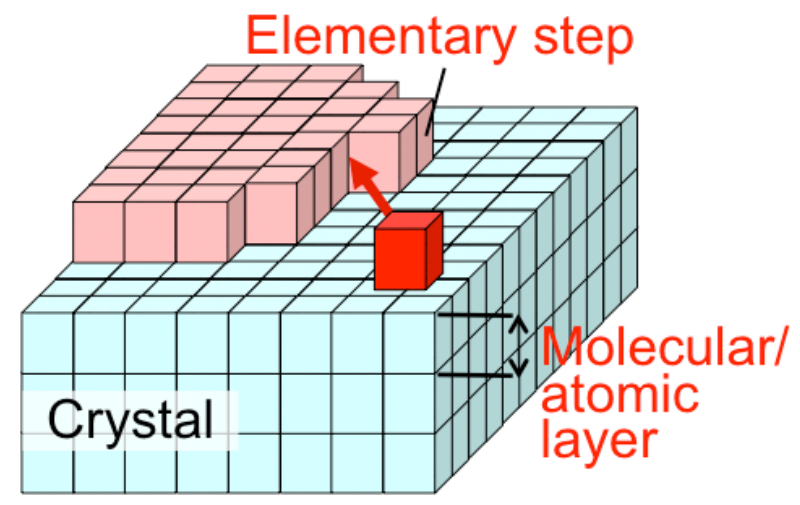

Fig. 1. A schematic illustration of the layer growth of a crystal.
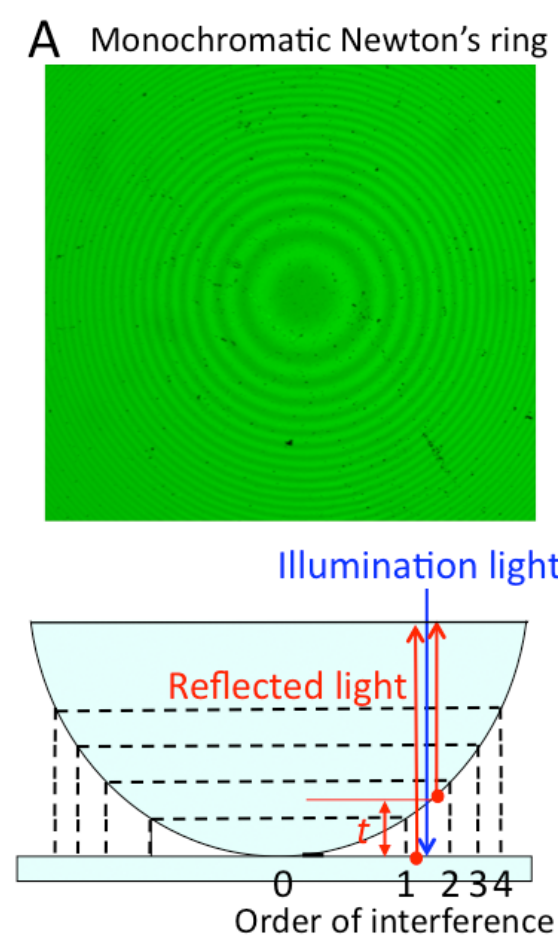

Condition for interference $R=2 t=i \cdot \lambda(i=0,1,2, .$.

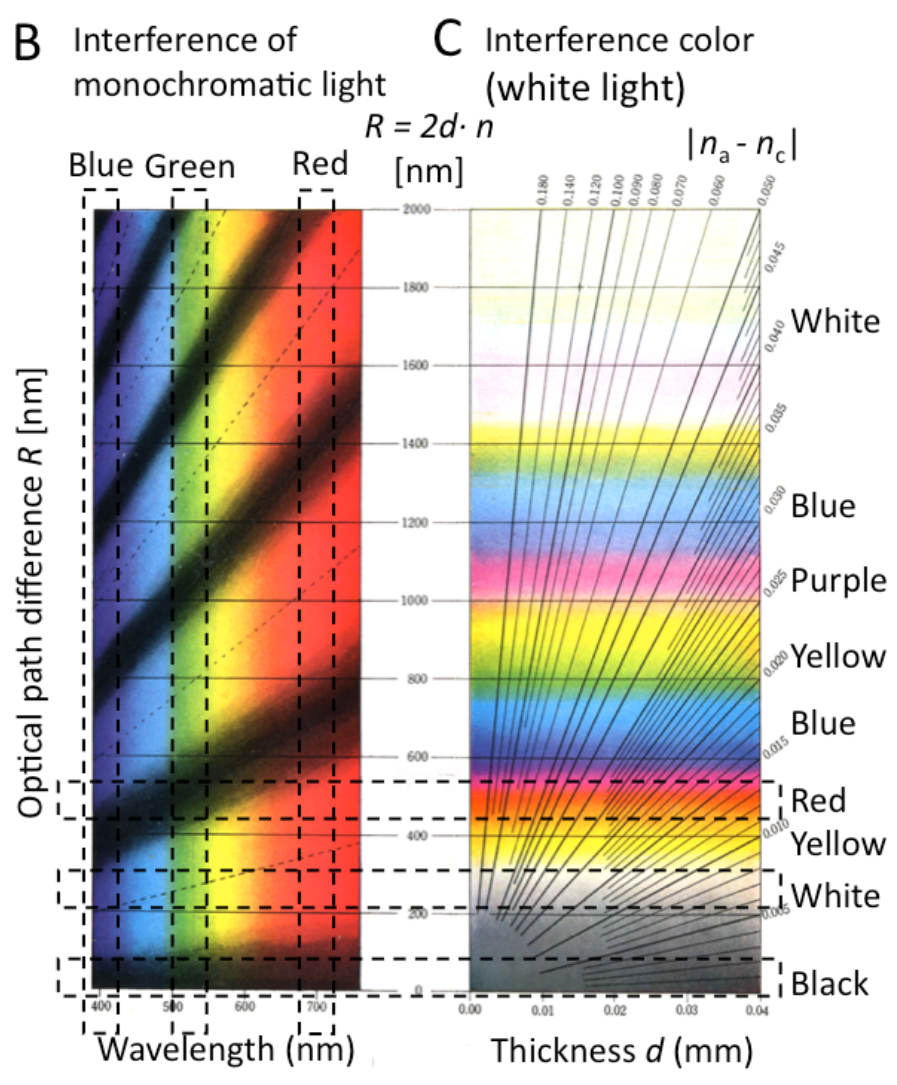

Fig. 2. Newton's rings formed by monochromatic light (A), interference of monochromatic light (B) and interference colors of white light (C). (A) A cross-sectional illustration of a setup is also shown. $\lambda$ is a wavelength, $t$ a height difference in a hemisphere glass, $R$ an optical path difference, and $i$ an interger. (B) $d$ is 
a thickness of a plate-like crystal and $n$ the refractive index of a plate-like crystal (C) $\left|n_{\mathrm{a}}-n_{\mathrm{c}}\right|$ shows an absolute value of the difference in refractive indexes in the $a$ - and $c$-directions. The color charts in B and C were taken from reference [6] and modified.

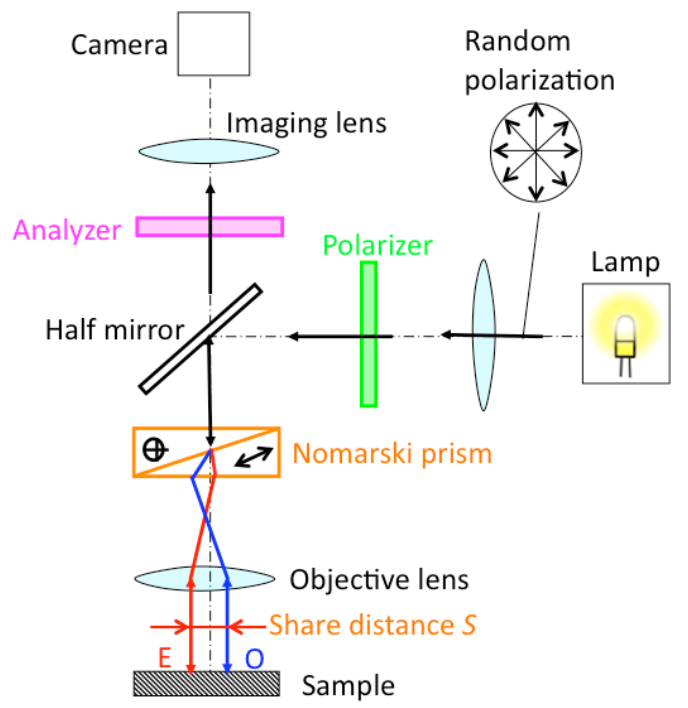

Fig. 3. A schematic optical path of a differential interference contrast microscope of the reflection type. 
A) Illumination light beam

Wave front

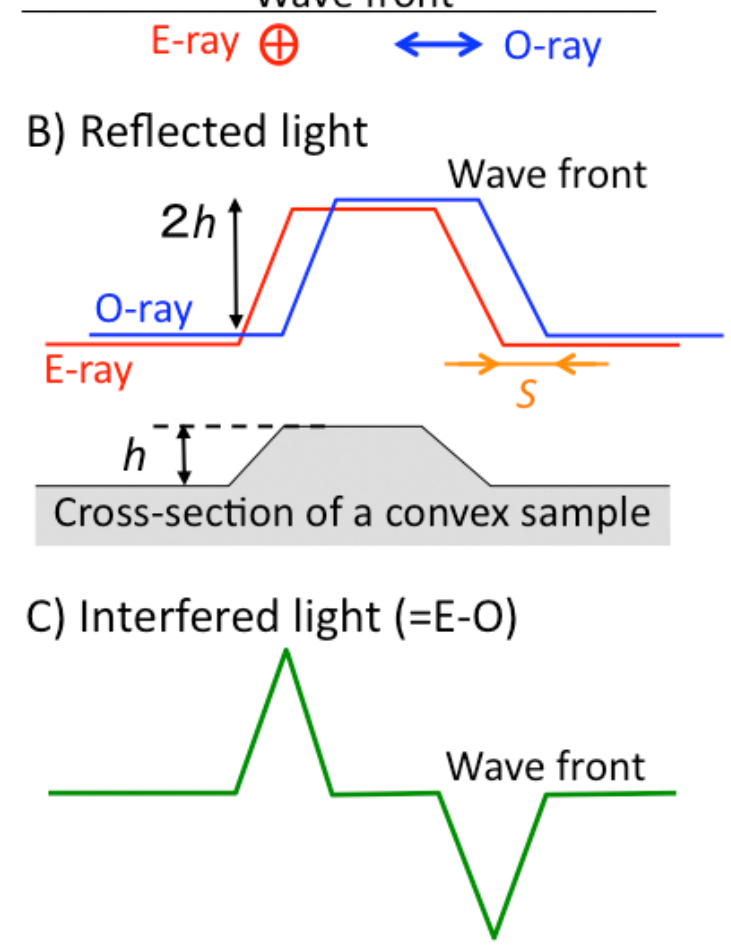

Fig. 4. Wave fronts of illumination light (A), light reflected from a convex sample surface (B), and interfered light (C). $h$ shows the height difference of a convex sample surface and $s$ a displacement distance.

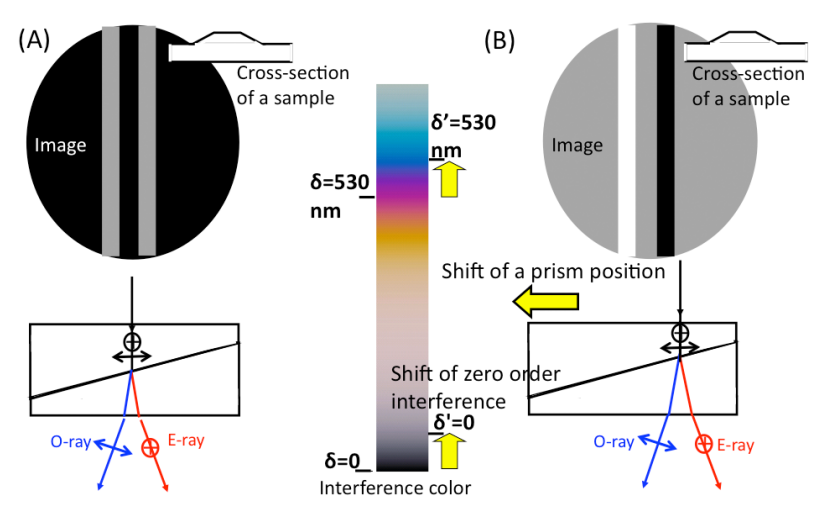

Fig. 5. Differential interference contrast. (A) Optical paths of O- and E-rays in a Nomarski prism are the same. (B) An optical path of an E-ray in a Nomarski prism is longer by $2 \delta$ than that of an O-ray. 

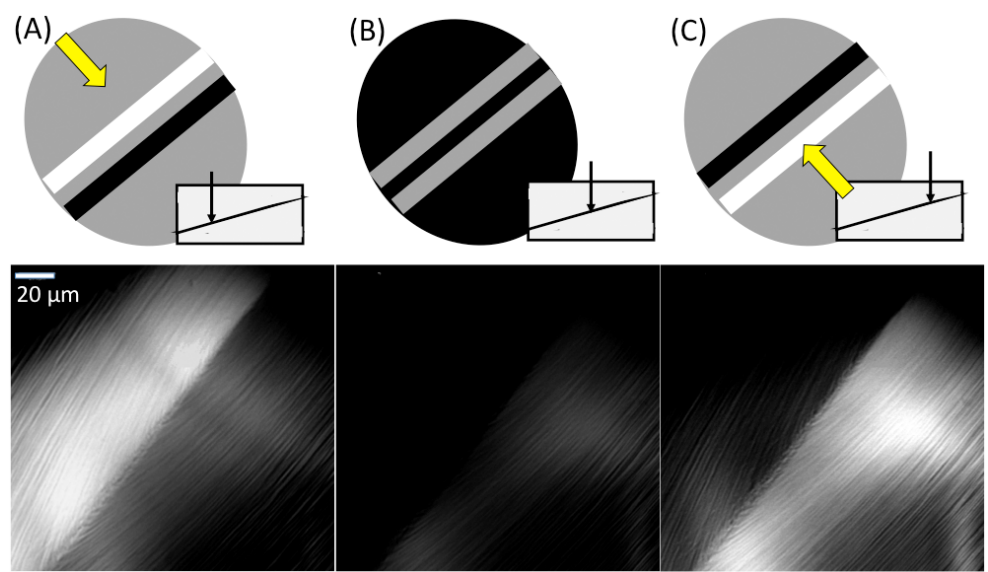

Fig. 6. Differential interference contrast microscopy images of a spiral growth hillock on a tetragonal crystal of hen egg-white lysozyme (model protein). The spiral growth hillock was visualized as if the hillock was illuminated from upper-left (A), above (B) and lower-right $(\mathrm{C})$ directions.
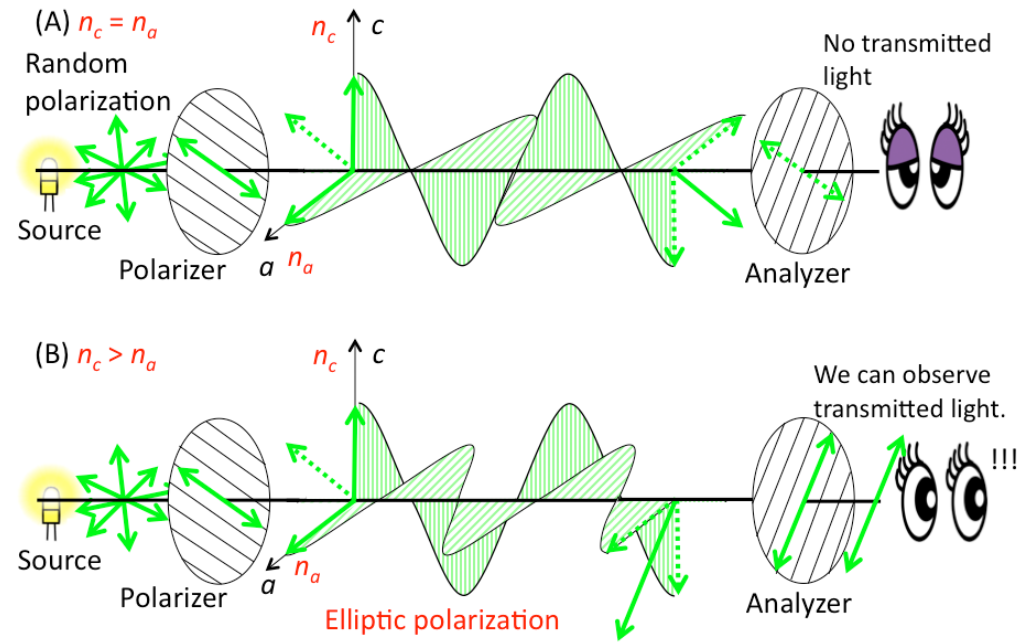

Fig. 7. Polarizing microscopy observation of isotropic (A) and anisotropic (B) samples. (A) Refractive indexes in $a$ - and $c$-directions that are normal to each other are the same. No rotation of the polarized right occurs. (B) The refractive index in the $c$-direction is larger than that in the $a$-direction. The direction of the transmitted polarized light rotates as the polarized light travels in the sample. 


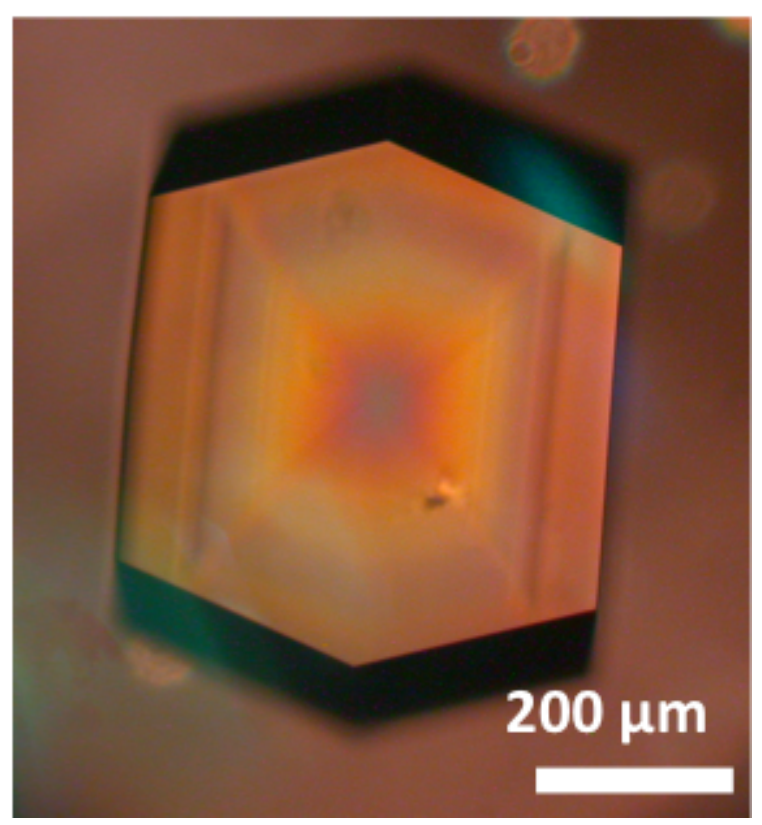

Fig. 8. A polarizing microscopy image of a tetragonal crystal of hen egg-white lysozyme (model protein). Directions of the polarizer and analyzer were normal to each other. After the tetragonal crystal was rotated until the whole crystal exhibited a dark contast, the photograph was taken with a long-time exposure (30-60s). 